\title{
Liquid biopsy for early stage lung cancer
}

\author{
Wenhua Liang*, Yi Zhao*, Weizhe Huang*, Hengrui Liang, Haikang Zeng, Jianxing He \\ Department of Thoracic Surgery and Oncology, the First Affiliated Hospital of Guangzhou Medical University, China State Key Laboratory of \\ Respiratory Disease \& National Clinical Research Center for Respiratory Disease, Guangzhou 510120, China \\ Contributions: (I) Conception and design: W Liang, J He; (II) Administrative support: Y Zhao, H Liang; (III) Provision of study materials or patients: \\ W Liang, Y Zhao, H Zeng; (IV) Collection and assembly of data: W Huang, Y Zhao; (V) Data analysis and interpretation: W Liang, H Liang; (VI) \\ Manuscript writing: All authors; (VII) Final approval of manuscript: All authors. \\ *These authors contributed equally to this work. \\ Correspondence to: Jianxing He. Department of Thoracic Surgery and Oncology, The First Affiliated Hospital of Guangzhou Medical University, State \\ Key Laboratory of Respiratory Diseases, National Clinical Research Center for Respiratory Center for Respiratory Disease, Guangzhou 510120 , \\ China. Email: drjianxing.he@gmail.com.
}

\begin{abstract}
Liquid biopsy, which analyzes biological fluids especially blood specimen to detect and quantify circulating cancer biomarkers, have been rapidly introduced and represents a promising potency in clinical practice of lung cancer diagnosis and prognosis. Unlike conventional tissue biopsy, liquid biopsy is noninvasive, safe, simple in procedure, and is not influenced by manipulators' skills. Notably, some circulating cancer biomarkers are already detectable in disease with low-burden, making liquid biopsy feasible in detecting early stage lung cancer. In this review, we described a landscape of different liquid biopsy methods by highlighting the rationale and advantages, accessing the value of various circulating biomarkers and discussing their possible future development in the detection of early lung cancer.
\end{abstract}

Keywords: Liquid biopsy; lung cancer; biomarkers; early detection; screening

Submitted Mar 22, 2018. Accepted for publication Mar 30, 2018.

doi: $10.21037 /$ jtd.2018.04.26

View this article at: http://dx.doi.org/10.21037/jtd.2018.04.26

\section{The importance of liquid biopsy for early stage lung cancer}

Lung cancer remains the most frequently diagnosed cancer and the leading cause of cancer-related mortality worldwide (1). In terms of its 5 -year survival rate which decreases as disease stage increases, there was a distinct range from less than $5 \%$ in stage $\mathrm{IV}$ to over $70 \%$ in stage I and specially, almost $100 \%$ for adenocarcinoma in situ (AIS) and minimally invasive adenocarcinoma (MIA) $(2,3)$. However, approximately $57 \%$ of patients present lung cancer at an advanced stage with metastasis at diagnosis (4). Therefore, it is imperative to identify diagnostic methods for early detection of lung cancer to differentiate patients from healthy populations, which enables a timely treatment at an initial stage of the disease and saves healthcare costs as well.

Since the National Lung Screening Trial (NLST) demonstrated a $20 \%$ mortality rate reduction in patients who had undergone chest low-dose computed tomography (LDCT) screening, compared to patients screened with a conventional chest X-ray (5), LDCT has been taken as a routine method for lung cancer screening. However, some limitations exist (5-9): (I) LDCT has a high false-positive rate, causing excessive medical care and unnecessary psychological burden; (II) LDCT is associated with repeated radiation exposure. NLST revealed the association of LDCT with the development of radiation-induced lung cancer; (III) LDCT is difficult to be integrated with early diagnosis approaches of other tumors. Known as using a routine blood draw and capturing tumor-related information from the blood by various techniques, liquid biopsy has significant potential to make up for the limitations of the traditional tissue-derived biomaterials obtained by surgery or needle biopsy and to make huge clinical practice in the detection of early lung cancer with 
the significant benefits as follows: non-invasive, easily and objectively accessible, and can be performed repeatedly.

\section{Biomarkers for the detection of early lung cancer}

To date, varieties of circulating cancer biomarkers are available for liquid biopsy including tumor-associated antigens (TAAs) (10), tumor-associated autoantibodies (TAAbs) (11), circulating tumor cells (CTCs) (12), circulating tumor DNA (ctDNA) (13), microRNA (miRNA) (14), exosomes (15) and so on. Herein, we present the rationale and characteristics of these markers.

\section{TAAs and autoantibodies (TAAbs)}

Due to the rarity of TAAs released into the circulation from early lung cancer and at the same time one or several markers are difficult to fully cover the various and heterogeneous lung cancer, conventional lung TAA markers detectable in serum like carcinoembryonic antigen (CEA), carbohydrate antigen (CA) 125, CA199, neuron specific enolase (NSE), cytokeratin 19 fragment 21-1 (Cyfra21-1) and squamous cell carcinoma (SCC) are hard to be used in detection of early lung cancer with poor sensitivity and specificity (16). They are more frequently used in the auxiliary diagnosis and the evaluation of curative effect.

However, serum TAAbs, autoantibodies against overexpressed, mutated, misfolded, or aberrant autologous cellular antigens, may be associated with unique advantages in identifying individuals with early lung cancer (16). That has been theoretically supported for reasons, which include: (I) immunosurveillance occurs in the early phase of cancer immuno-editing process (17) and hence autoantibodies may be detectable in early stage of lung cancer $(11,18)$; (II) TAAbs can be present at high titers even tumor mass is low and are stable in blood. Detection using a panel of 7-AABs (19), including p53, GAGE7, PGP9.5, CAGE, MAGEA1, SOX2 and GBU4-5, was the first approved liquid biopsy approach by China Food and Drug Administration (CFDA) for helping to distinguish lung nodules. Their concentrations could be quantitated by enzyme-linked immunosorbent assay (ELISA), which is relatively low cost and easy-toperform. Therefore, detection based on this AABs panel hold promise for detection of early lung cancer.

\section{CTCs}

CTCs are cancer cells directly shed off from primary tumor sites and/or metastatic sites and float in the circulation, which can be isolated as either single cells or clusters. These cancer cells usually undergo epithelial-mesenchymal transition (EMT) and become invasive and motile after detaching from epithelial sheets. They migrate into the bloodstream by overcoming the vessel walls to which they normally adhere, in which way they become CTCs, and then invade distant sites and proliferate $(20,21)$. CellSearch technology, the first and only U.S. Food and Drug Administration (FDA) approved CTC detection system, has been cleared for clinical CTC detection in metastatic prostate, breast and colorectal cancers (22-25). However, their low concentrations in blood (1-10 CTCs per $10 \mathrm{~mL}$ ) (26) and lack of cancer-specific surface markers (27) (especially after EMT) make a low early identify rate, therefore, posing serious challenges in application of CTC detection in early diagnosis. A multitude of enrichment technologies have been developed, the CTC-based Folate Receptor PCR, for instance, providing an increase in sensitivity but is limited to adenocarcinoma types $(12,28)$. To date, the clear clinical application of CTC detection is still under investigation and it provides potential application in intracellular pathology diagnosis and as a prognostic marker.

\section{$c t D N A$}

ctDNA is cell-free fragments of DNA shed into the bloodstream by tumor cells undergoing necrosis, apoptotic or active secretion events (29). It is tumor specific and provides molecular clues about fragmented DNAs of tumor cells and their specific mutations. Quantitative and qualitative analysis respectively on the amount and biological characteristics of ctDNA provide real-time evaluations for diagnostic and prognostic assessments. Firstly, in general, the amount of normal circulating cfDNA is significantly higher in patients with tumors than that in patients who are healthy or with benign diseases (30-35). Furthermore, the amount of ctDNA was demonstrated to be associated with tumor burden, tumor response, and survival outcome $(36,37)$. Secondly, with the same genomic alterations that are present in the corresponding tumor, ctDNA has biological characteristics containing the information such as genetic mutation, fusion, deletion, insertion, rearrangement, methylation and other forms of tumor-specific such as abnormalities microsatellite instability (MSI) and loss of heterozygosity (LOH) (38). Therefore, ctDNA might be a highly promising biomarker 
in the detection of early lung cancer, response to therapy, emerging drug resistance mechanisms and relapse.

In recent years, our team has been trying to detect the presence of small lesions in patients with lung cancer by chip capturing and high-throughput sequencing. Using this method, we previously conducted two studies. In the first study, we screened out the most important genetic mutations in Asian population based on a large-sample next generation sequencing (NGS) based profiling (39). Using this information, a panel of the most frequent gene mutation that could cover more than $80 \%$ of mutation sites was designed. Subsequently, we recruited 38 patients with suspected lung nodules which were diagnosed to be malignant in 34 patients according to the histopathological findings. For all patients, we performed ultra-deep sequencing $(>30,000 \times$ on average) to screen lung cancerassociated mutations in both formalin-fixed paraffin embedded (FFPE) samples for tissue biopsies and matched blood plasma samples for cfDNA detecting. The results of tissue biopsies revealed no mutations were present in the 4 patients with benign nodules and 157 mutations in 32 patients with Lung adenocarcinoma were consistent with the Catalogue of Somatic Mutations in Cancer (COSMIC) database, in which 76 mutations were reported under cfDNA measurements in 12 patients. Only 6 identical mutations were present in both tissue-derived DNA and plasma-derived cfDNA mutation profile. We established an algorithm, showing that the sensitivity is only $33 \%$ with $100 \%$ specificity (40).

In the other study, we performed high-throughput DNA sequencing using Illumina 450k Bead Methylation chip array in lung cancer tissue samples to identify cancer-specific methylation sites, and then match ctDNA methylation signatures in the corresponding plasma sample. From an independent validation set of 129 plasma samples in 63 patients with malignant lung nodules and 66 patients with benign nodules, we were able to achieve a sensitivity of $82.5 \%$ for identification of malignant nodules, with a specificity of $83.3 \%$. Specifically, our assay is demonstrated to be highly sensitive towards early-stage lung cancer detection, with a sensitivity of $81.5 \%$ in a total of 27 patients with stage Ia lung cancer (41). To the best of our knowledge, this ctDNA methylation-based diagnostic assay is the most sensitive tool for identification of early stage lung cancer to date and a large-scale multicenter study is undertaken to validate its clinical value in early detection of lung cancer.

\section{Other significant biomarkers}

There is some other source of liquid biopsy-based biomarkers can be considered for early detection of lung cancer. Exosomes, which could be secreted by either normal or tumor cells, are extracellular nanosized vesicles in spherical shape with a diameter of 30-100 nm (42). Normal cell-derived exosomes play a role in maintaining stable homeostasis (43), whereas tumor cell-derived exosomes are related to tumor progression, such as ensuring the growth and survival of tumor cells, helping in their escape from immune surveillance and guiding their metastasis and proliferation (44-47). In general, Exosomes contain various nuclear acids, proteins and lipids (15), which provide a possibility that exosomes could be used as a diagnostic biomarker for lung cancer with the help of specific extraction techniques. MiRNAs are small noncoding RNA gene products in a length of about 22 nucleotides and have a key role in various biological processes through the regulation of gene expression (48). A significantly difference was observed between primary lung cancers and corresponding normal lung tissues from the genome-wide expression profiling analysis of miRNAs (49). Additionally, different cancers are associated with different miRNAs expression and such as the Let-7 family are novel biomarkers for lung cancer (50). With other characteristics such as usually highly stable in blood (51), miRNAs may become ideal biomarkers for lung cancer detection.

\section{Combination of biomarkers}

The liquid biopsy-based biomarkers we described above contain abundant genomic, proteomic and other information reflecting the tumor progression. As lung cancer is the most heterogeneous cancer in terms of genesis and progression, detecting single type of these biomarkers is limited by low coverage of all types of lung cancer. The limitations are likely to be overcome by combining different markers with relatively high detection efficiency to make an improvement in sensitivity and specificity of lung cancer diagnosis. Researchers from Johns Hopkins University (JHU) published their latest research on Science (52), they developed a liquid biopsy called CancerSEEK that can simultaneously detect eight common cancer types at their early stage through assessment of the levels of 8 circulating proteins and 16 mutations in cell-free DNA. In addition, another recent research (53) published on Annals of Oncology focused on EGFR mutation detection in NSCLC patient 
plasma where exosomal RNA and ctDNA (exoNA) were co-isolated using ExoLution ${ }^{\mathrm{TM}}$ Plus extraction technology (Exosome Diagnostics Inc.). An increased sensitivity was obtained when using exoRNA compared with only ctDNA for detection. In terms of our previous work on mutation and methylation detection, we found that there was no obvious co-occurring pattern between the major driver mutations and methylations, suggesting that the detection of genetic mutations and methylations could complement each other in diagnosing the early lung cancer (data not shown). Such codetection field is currently under investigation.

\section{Applications of liquid biopsy for early lung cancer}

The immediate clinical applications of liquid biopsy for early lung cancer are disease auxiliary diagnosis and screening.

Firstly, once suspicious lesions were found by CT scan, the identification of their properties could be further confirmed by liquid biopsy (54). Considering the cost, most of studies at present focus on the value of liquid biopsy used as an auxiliary diagnosis tool to improve CT scan effectiveness, however, the advanced development of artificial intelligence in medical care may rapidly blur such necessity. Secondly and notably, liquid biopsy is developing with an aim to replace CT scan as a new lung cancer screening approach, because biomarkers present early enough in the bloodstream, even before the presence of abnormal images, and are detectable for the diagnosis of early stage lung cancer. TracerX study (55) concluded that up to 6-12 months was earlier in lung cancer diagnosis using specific and sensitive ctDNA than using imaging techniques. In addition, CT screening results in a rate of overdiagnosis and radiation risk and raised concern on the its real cost-effectiveness.

At present, the associated TAABs are the only available biomarkers for large-scale lung cancer studies done in governmental screening settings. A randomized controlled trial, the biggest lung cancer screening program involving 12,000 participants in Scotland with high risk of developing lung cancer, was conducted based on a panel of 7-AABs (56). It revealed a specificity of $93 \%$ for the EarlyCDT-lung test detecting autoantibodies to proteins in the earliest stages of lung cancer.

\section{The future development of liquid biopsy}

Leaving out the cancer-related genetic and environmental factors, we have no more information on what kind cancer people may develop. At this point, the integration of screening service including all canners should be highlighted in health care system. Liquid biopsy, as one of the contributors, will be developed into more advance in correct qualitative diagnosis and causative localization. DNA methylation has showed a considerable localization feature (57). Furthermore, the nucleosomes in cfDNA carry some genetic information of the cells from which they originated and can be utilized for qualitative diagnosis (58). There are several companies have committed to the development of whole-cancer screening by liquid biopsy. For instance, the company GRAIL, spun out from Illumina, attracted founds from Google, Microsoft and Amazon and raised $\$ 1$ billion in total to develop its technology of cancer blood detecting. In 2016, its plan of whole-cancer screening was launched to promote the process of early cancer detection (potentially before a tumor or any symptoms are noticeable) using blood samples and would be generalized in the later 3 years. The blood detection of lung cancer is just one of the branches.

\section{Summary}

We have reviewed the current state of liquid biopsy for lung cancer and provide an outlook of its clinical utility. With a wide variety of promising biomarkers, liquid biopsy for lung cancer can generate valuable information about cancer genetic characteristics almost in real time. We believe this non-invasive technology can be generally used as a key tool for detection of early lung cancer and even replace CT scan in lung cancer screening. Further efforts are warranted, including transomics analysis and validation studies.

\section{Acknowledgements}

Funding: Chinese National Natural Science Foundation (Grant No. 81501996); Key Project of Guangzhou Scientific Research Project (Grant No. 201804020030); Guangdong Doctoral Launching Program (Grant No. 2014A030310460); Doctoral Launching Program of Guangzhou Medical University (Grant No. 2014C27); and Key Project of Livelihood Technology of Guangzhou (2011Y2-00024).

\section{Footnote}

Conflicts of Interest: The authors have no conflicts of interest 
to declare.

\section{References}

1. Ferlay J, Soerjomataram I, Dikshit R, et al. Cancer incidence and mortality worldwide: sources, methods and major patterns in GLOBOCAN 2012. Int J Cancer 2015;136:E359-86.

2. Siegel RL, Miller KD, Jemal A. Cancer statistics, 2015. CA Cancer J Clin 2015;65:5-29.

3. Goldstraw P. The 7th Edition of TNM for Lung and Pleural Tumours. Journal of Clinical and Analytical Medicine 2012;3:123-7.

4. Patz EF, Jr., Campa MJ, Gottlin EB, et al. Panel of serum biomarkers for the diagnosis of lung cancer. J Clin Oncol 2007;25:5578-83.

5. Ostrowski M, Marjanski T, Rzyman W. Low-dose computed tomography screening reduces lung cancer mortality. Adv Med Sci 2018;63:230-6.

6. David DJ, Brenner EJ. Computed Tomography - An Increasing Source of Radiation Exposure. $\mathrm{N}$ Engl J Med 2007;357:2277-84.

7. Brenner DJ, Hall EJ. Computed tomography--an increasing source of radiation exposure. $\mathrm{N}$ Engl J Med 2007;357:2277-84.

8. Berrington de Gonzalez A, Mahesh M, Kim KP, et al. Projected cancer risks from computed tomographic scans performed in the United States in 2007. Arch Intern Med 2009;169:2071-7.

9. Croswell JM, Baker SG, Marcus PM, et al. Cumulative incidence of false-positive test results in lung cancer screening: a randomized trial. Ann Intern Med 2010;152:505-12, W176-80.

10. Tarro G, Perna A, Esposito C. Early diagnosis of lung cancer by detection of tumor liberated protein. J Cell Physiol 2005;203:1-5.

11. Chapman CJ, Murray A, McElveen JE, et al. Autoantibodies in lung cancer: possibilities for early detection and subsequent cure. Thorax 2008;63:228-33.

12. Chen X, Zhou F, Li X, et al. Folate Receptor-Positive Circulating Tumor Cell Detected by LT-PCR-Based Method as a Diagnostic Biomarker for Non-Small-Cell Lung Cancer. J Thorac Oncol 2015;10:1163-71.

13. Diaz LA, Jr., Bardelli A. Liquid biopsies: genotyping circulating tumor DNA. J Clin Oncol 2014;32:579-86.

14. Zandberga E, Kozirovskis V, Abols A, et al. Cell-free microRNAs as diagnostic, prognostic, and predictive biomarkers for lung cancer. Genes Chromosomes Cancer 2013;52:356-69.
15. Cui S, Cheng Z, Qin W, et al. Exosomes as a liquid biopsy for lung cancer. Lung Cancer 2018;116:46-54.

16. Murray A, Chapman CJ, Healey G, et al. Technical validation of an autoantibody test for lung cancer. Ann Oncol 2010;21:1687-93.

17. FM B. The concept of immunological surveillance. Prog Exp Tumor Res 1970;13:1-27.

18. Jett JR, Peek LJ, Fredericks L, et al. Audit of the autoantibody test, EarlyCDT(R)-lung, in 1600 patients: an evaluation of its performance in routine clinical practice. Lung Cancer 2014;83:51-5.

19. Ren S, Zhang S, Jiang T, et al. Early detection of lung cancer by using an autoantibody panel in Chinese population. Oncoimmunology 2017;7:e1384108.

20. Plaks V, Koopman CD, Werb Z. Cancer. Circulating tumor cells. Science 2013;341:1186-8.

21. Satelli A, Mitra A, Brownlee Z, et al. Epithelialmesenchymal transitioned circulating tumor cells capture for detecting tumor progression. Clin Cancer Res 2015;21:899-906.

22. Krebs MG, Sloane R, Priest L, et al. Evaluation and prognostic significance of circulating tumor cells in patients with non-small-cell lung cancer. J Clin Oncol 2011;29:1556-63.

23. Aggarwal C, Meropol NJ, Punt CJ, et al. Relationship among circulating tumor cells, CEA and overall survival in patients with metastatic colorectal cancer. Ann Oncol 2013;24:420-8.

24. Deneve E, Riethdorf S, Ramos J, et al. Capture of viable circulating tumor cells in the liver of colorectal cancer patients. Clin Chem 2013;59:1384-92.

25. Cristofanilli M, Budd GT, Ellis MJ, et al. Circulating tumor cells, disease progression, and survival in metastatic breast cancer. N Engl J Med 2004;351:781-91.

26. Dharmasiri U, Witek MA, Adams AA, et al. Microsystems for the capture of low-abundance cells. Annu Rev Anal Chem (Palo Alto Calif) 2010;3:409-31.

27. Alix-Panabieres C, Pantel K. Circulating tumor cells: liquid biopsy of cancer. Clin Chem 2013;59:110-8.

28. Nunez MI, Behrens C, Woods DM, et al. High expression of folate receptor alpha in lung cancer correlates with adenocarcinoma histology and EGFR [corrected] mutation. J Thorac Oncol 2012;7:833-40.

29. Crowley E, Di Nicolantonio F, Loupakis F, et al. Liquid biopsy: monitoring cancer-genetics in the blood. Nat Rev Clin Oncol 2013;10:472-84.

30. Hashad D, Sorour A, Ghazal A, et al. Free circulating tumor DNA as a diagnostic marker for breast cancer. J Clin Lab Anal 2012;26:467-72.

31. No JH, Kim K, Park KH, et al. Cell-free DNA level 
as a prognostic biomarker for epithelial ovarian cancer. Anticancer Res 2012;32:3467-71.

32. Park JL, Kim HJ, Choi BY, et al. Quantitative analysis of cell-free DNA in the plasma of gastric cancer patients. Oncol Lett 2012;3:921-6.

33. Salvianti F, Pinzani P, Verderio P, et al. Multiparametric analysis of cell-free DNA in melanoma patients. PLoS One 2012;7:e49843.

34. Schwarzenbach H, Muller V, Milde-Langosch K, et al. Evaluation of cell-free tumour DNA and RNA in patients with breast cancer and benign breast disease. Mol Biosyst 2011;7:2848-54.

35. Schwarzenbach H, Stoehlmacher J, Pantel K, et al. Detection and monitoring of cell-free DNA in blood of patients with colorectal cancer. Ann N Y Acad Sci 2008;1137:190-6.

36. Brevet M, Johnson ML, Azzoli CG, et al. Detection of EGFR mutations in plasma DNA from lung cancer patients by mass spectrometry genotyping is predictive of tumor EGFR status and response to EGFR inhibitors. Lung Cancer 2011;73:96-102.

37. Kimura H, Suminoe M, Kasahara K, et al. Evaluation of epidermal growth factor receptor mutation status in serum DNA as a predictor of response to gefitinib (IRESSA). British Journal of Cancer 2007;97:778-84.

38. Fleischhacker M, Schmidt B. Circulating nucleic acids (CNAs) and cancer--a survey. Biochim Biophys Acta 2007;1775:181-232.

39. Wu K, Zhang X, Li F, et al. Frequent alterations in cytoskeleton remodelling genes in primary and metastatic lung adenocarcinomas. Nat Commun 2015;6:10131.

40. Ye M, Li S, Huang W, et al. Comprehensive targeted super-deep next generation sequencing enhances differential diagnosis of solitary pulmonary nodules. J Thorac Dis 2018:10:S820-S829.

41. Liang $W$, Zhao $Y$, Huang $W$, et al. Non-invasive diagnosis of solitary pulmonary nodules using high-throughput targeted DNA methylation sequencing of circulating tumor DNA. J Thorac Oncol 2017;12:S2240.

42. Taverna S, Giallombardo M, Gil-Bazo I, et al. Exosomes isolation and characterization in serum is feasible in non-small cell lung cancer patients: critical analysis of evidence and potential role in clinical practice. Oncotarget 2016;7:28748-60.

43. Vyas N, Dhawan J. Exosomes: mobile platforms for targeted and synergistic signaling across cell boundaries. Cell Mol Life Sci 2017;74:1567-76.

44. Melo SA, Luecke LB, Kahlert C, et al. Glypican-1 identifies cancer exosomes and detects early pancreatic cancer. Nature 2015;523:177-82.
45. Kahlert C, Kalluri R. Exosomes in tumor microenvironment influence cancer progression and metastasis. J Mol Med (Berl) 2013;91:431-7.

46. Hoshino D, Kirkbride KC, Costello K, et al. Exosome secretion is enhanced by invadopodia and drives invasive behavior. Cell Rep 2013;5:1159-68.

47. Zhang L, Zhang S, Yao J, et al. Microenvironmentinduced PTEN loss by exosomal microRNA primes brain metastasis outgrowth. Nature 2015;527:100-4.

48. Ambros V. MicroRNA Pathways in Flies and Worms. Cell 2003;113:673-6.

49. Yanaihara N, Caplen N, Bowman E, et al. Unique microRNA molecular profiles in lung cancer diagnosis and prognosis. Cancer Cell 2006;9:189-98.

50. Xie P, Li X, Tan X, et al. Sequential Serum Let-7 Is a Novel Biomarker to Predict Accelerated Reproliferation During Fractional Radiotherapy in Lung Cancer. Clin Lung Cancer 2016;17:e95-e101.

51. Mitchell PS, Parkin RK, Kroh EM, et al. Circulating microRNAs as stable blood-based markers for cancer detection. Proc Natl Acad Sci U S A 2008;105:10513-8.

52. Cohen JD, Li L, Wang Y, et al. Detection and localization of surgically resectable cancers with a multi-analyte blood test. Science 2018;359:926-30.

53. Krug AK, Enderle D, Karlovich C, et al. Improved EGFR mutation detection using combined exosomal RNA and circulating tumor DNA in NSCLC patient plasma. Ann Oncol 2018;29:700-6.

54. Giulia Veronesi FB, Maurizio Infante, Marco Alloisio. The challenge of small lung nodules identified in CT screening: can biomarkers assist diagnosis? Biomark Med 2016;10:137-43.

55. Jamal-Hanjani M, Wilson GA, McGranahan N, et al. Tracking the Evolution of Non-Small-Cell Lung Cancer. N Engl J Med 2017;376:2109-21.

56. Sullivan F, Dorward A, Mair F, et al. P2.06-038 An RCT of the Detection of Autoantibodies to Tumor Antigens in Lung Cancer Using the EarlyCDT-Lung Test in Scotland (ECLS) in 12208 Study Subjects. J Thorac Oncol 2017;12:S1095.

57. Laird PW. The power and the promise of DNA methylation markers. Nat Rev Cancer 2003;3:253-66.

58. Sharma S, Kelly TK, Jones PA. Epigenetics in cancer. Carcinogenesis 2010;31:27-36.

Cite this article as: Liang W, Zhao Y, Huang W, Liang H, Zeng H, He J. Liquid biopsy for early stage lung cancer. J Thorac Dis 2018;10(Suppl 7):S876-S881. doi: 10.21037/ jtd.2018.04.26 